\title{
CAUSE AND EFFECT ANALYSIS IN CHEMICAL PROCESSES UTILIZING XML, PLANT CONNECTIVITY AND QUANTITATIVE PROCESS HISTORY
}

\author{
Jegatheeswaran Thambirajah*, Lamia Benabbas ${ }^{\mathrm{x}}$, Margret Bauer ${ }^{+}$and Nina F Thornhill* ${ }^{0}$ \\ *Centre for Process Systems Engineering, Department of Chemical Engineering, Imperial College \\ London, London SW7 2AZ, UK \\ ${ }^{\mathrm{x}} \mathrm{ABB}$ Strategic R\&D for Oil and Gas, Ole Deviks Vei 10, 0666 Oslo, Norway \\ ${ }^{+}$ABB Corporate Research, Wallstadter Strasse 59, D-68526 Ladenburg, Germany \\ ${ }^{0}$ Author for correspondence: Tel: + 44 (0)20 7594 6622; E-mail: n.thornhill@imperial.ac.uk
}

\begin{abstract}
Disturbances that spread plant-wide in a chemical process pose challenges to maintenance staff. Connections within the plant and the presence of multiple causal paths mean it is not straightforward to locate the root disturbance because the effects can propagate and be detected elsewhere. Measurementbased methods use quantitative process history to generate hypotheses about the root cause, while a separate strand of work in the literature has used causal maps and digraphs. It has been reported that both approaches can give spurious solutions, however. The idea behind this article is to reduce the number of spurious solutions by combining basic and readily-available information about the connectivity of the process with the results from causal measurement-based analysis. Connectivity information is captured from an XML description of the process schematic that complies with the CAEX schema. The capabilities of the approach and its potential for future development are discussed.
\end{abstract}

Keywords: Connectivity matrix; fault detection and diagnosis, plantwide disturbance; plant topology; root cause; XML.

\section{Introduction}

Several authors (Chiang and Braatz, 2003; Lee et al., 2003) have suggested it is possible to develop better techniques of fault diagnosis by combining a qualitative method with a quantitative one. This paper presents an approach that generates hypotheses regarding the possible root cause of a plant-wide disturbance using statistical measurement-based analysis, and tests the hypotheses by automated examination of the connectivity of the process using graph traversal. A measurement-based method greatly narrows down the search for the root cause of a disturbance, but may indicate more than one candidate root cause for a single disturbance. Additional information about the process that is provided by a causal model in the form of a connectivity matrix allows further testing of the measurement-based hypotheses and identifies the candidate that can best explain all other observations.

The core technology for the extraction of a connectivity matrix is a description in XML (eXtensible Markup Language) of the process schematic. XML facilitates the sharing of data across different systems. It is well established in e-commerce, for instance the Chem eStandards ${ }^{\mathrm{TM}}$ for the buying, selling and delivery of chemical products use XML for electronic data exchange, while Varma et al. (2003) used an XML-based language to represent data, logical relationships and precedence constraints for managing new product development projects which compete for limited resources. These examples show that XML is useful for allowing different users and applications to share data in chemical engineering applications. XML has also been used for management and manipulation of suites of models (Hetherington et al., 2007; Lam et al, 2007), while the release 3.0 of Aspentech's HYSYS has an XML infrastructure for management of the reuse and interoperation of simulations. The AEX project of the FIATECH consortium (Palmer, 2007) is generating XML specifications for engineering equipment such as pumps to facilitate design and procurement of large engineering projects. FIATECH is also engaged in activity towards deployment of ISO 15926, a standard for engineering data exchange.

XML has recently started to be used in intelligent P\&ID tools for export and exchange of process drawings and piping and instrumentation diagrams (P\&IDs) e.g. in Comos P\&ID from Siemens, SmartPlant P\&ID from Intergraph and Aveva's VPE P\&ID. Process topology can be exported from these tools as open XML text that describes equipment items, their properties, the connections between them and the directions of those connections. Computer Aided Engineering Exchange (CAEX) and XMpLant are two relevant XML schemas. Fedai and Drath (2005) described a representation of a process diagram which conforms to the CAEX schema as specified in IEC/PAS 62424. Leal (2005) gave a detailed description of the ISO 10303-221 and ISO 15926 standards for engineering data exchange, for which XMpLant (Laud, 2006) provides a schema. An XML text file that complies with one of these schemas provides a basic, low cost qualitative process description which can be readily generated from a process drawing or P\&ID. Academic publications to date include automatic generation of asset monitors and safety interlock codes (Schmidberger et al. 2006; Drath et. al, 2006) which both used CAEX. 
This paper explains the derivation of a connectivity matrix from a CAEX representation of the process diagram. Causal analysis based on process measurements as described in a previous paper (Bauer et al., 2007) provides an indication of the dynamic relationships between measurement points and suggests possible root causes of a plant-wide disturbance. Candidate hypotheses from the causal analysis about the source of the disturbance are then tested for consistency with the connectivity matrix. For instance, a root cause hypothesis is reinforced if there is a feasible propagation path to all other locations where the disturbance has been detected. The root cause hypothesis that can explain the propagation of the disturbance to every one of the other points where it has been detected would indicate the most likely root cause of the disturbance.

Section 2 describes the background and context of the work and Section 3 explains the methods that were employed including: the representation of the process schematic in CAEX, the method used to derive the connectivity matrix, the generation of root cause hypotheses, and the graph traversal algorithm that was used for the purpose of propagation path determination. A demonstration of their integration is given in Section 4 using an industrial case study with a plant-wide disturbance. The demonstration examines each postulated root cause of the disturbance for consistency with the process connectivity and draws a conclusion about the most likely overall root cause. Section 5 briefly describes a second example in which more than one disturbance was present. As XML representations of processes are becoming more widely available some speculation on future development is timely. Section 6 gives a critical appraisal of the potential of a connectivity matrix to resolve ambiguities in the results from measurement-based analysis, and suggests some future enhancements. The article ends with a summary and conclusion.

\section{Background and context}

A chemical process consists of connected components and pipes through which utility streams and chemical reactants flow, together with controllers and monitoring instruments. A fault or disturbance may occur which manifests as a deviation in measurements, typically in flow, pressure, level or temperature. The connectivity of a continuous process means disturbances often propagate plant-wide.

In such a case, the use of human labour to pin-point the source of a disturbance which could have many origins becomes time-consuming, difficult and expensive. These considerations, together with reports of the economic losses resulting from abnormal situations (e.g. Nimmo, 1995; Laser, 2000) provide the motivation for a system that automates the process of searching for the root cause when an affected parameter is found.

\subsection{Methods using qualitative models}

The review series by Venkatasubramanian et al., (2003a,b,c) discussed methods for detection, isolation and diagnosis of faults in chemical processes, classifying the available methods into quantitative and qualitative model-based methods and quantitative and qualitative process history based methods.

Qualitative models for use in process fault diagnosis should capture causal relationships. As well as signed digraphs (SDG) (Venkatasubramanian et al., 2003b; Maurya et al., 2004; Srinivasan et al. 2006), other causal representations include Multilevel Flow Modelling (Petersen, 2000; Larsson, 2007), Bayesian belief networks (Weidl, et al., 2005), rule-based systems (e.g. Blue Circle Industries, 1990) and fault tree methods as used in the alarm management of safety critical systems (e.g. Vesely et al, 1981).

Maurya et al., $(2003 a, b)$ gave a review of graph-based approaches for safety analysis and diagnosis of chemical process systems and showed how to develop graph models systematically from a system of differential-algebraic equations. A connectivity matrix derived from a CAEX description is a simple causal qualitative model generated from a process schematic without the need for a first principles model. The connectivity matrix captures directional links between connected items of equipment and thus includes the same information as a directed graph. It cannot be used to generate a signed digraph, however, because it does not include information about whether the causal influence is positive or negative.

\subsection{Methods using process history}

Quantitative process history methods use measurements from the process (Venkatasubramanian et al., 2003c). They include multivariate statistical process analysis which is typically used for slowly developing faults such as catalyst degradation or fouling of a heat exchanger, qualitative trend analysis, and signal analysis methods for detection and diagnosis of persistent dynamic disturbances such as limit cycle oscillations.

Disturbance diagnosis is facilitated by multivariate methods which can infer causation from process measurements. Holland (1986) and Pearl (1995) have described how causal graphs can be estimated from data. Establishing cause-and-effect (rather than correlation alone) from experimental data is difficult. However, measurements from industrial operations are usually in the form of time series and causal direction can be inferred when information about time is available. Pair-wise testing of the time series of process measurements leads to a cause-and-effect matrix, which may also be represented as a directed graph. One way to conduct pair-wise testing uses estimates of conditional probabilities (a Bayesian network). Transfer entropy (Schreiber, 2000) adds information about time into a Bayesian network because it tests hypotheses concerning the joint and conditional probabilities of past and current values in a time series.

\subsection{Combination methods}

Several authors have recognized the benefits of combining models with data. Vedam and Venkatasubramanian (1999) and Chiang and Braatz (2003) showed enhanced diagnosis using measurement-based analysis if a qualitative model is available. Norvilas et al. (2000) combined multivariate statistical analysis and an expert system for the same purpose, while Leung and Romagnoli (2002) 
integrated a multivariate statistical analysis method with cause and effect map of a process, which was set up manually, to help in the diagnosis of faults. Lee et al., (2003) also combined SDGs with multivariate statistical analysis for enhanced diagnosis.

Maurya et. al (2007) have recently demonstrated linkage of SDG analysis with qualitative trend analysis. They observed that SDG methods when used alone for diagnosis can generate spurious solutions. The same is true of cause-and-effect analysis using process measurements, as is discussed later in this paper. Maurya and his co-authors noted that a causal model based on the process schematic will enhance a purely measurement-based analysis because it contains additional information. The implication is that by combining two different techniques, it is possible to reduce the number of spurious solutions that may arise.

\subsection{Contribution of the paper.}

The contribution of this paper is to demonstrate the benefits of combining the cause-and-effect matrix derived from measurements of a process, and qualitative information about the process layout in the form of a connectivity matrix.

The paper builds on the work of Yim et al., (2006). In that work, a list of elements in the process schematic and the connections between them were parsed from its CAEX description. A rule-base was then used to confirm the results of plant-wide oscillation detection and diagnosis results from an industrial case study. The advances reported here are: (i) generation of a process connectivity matrix from the CAEX description that lends itself to automated, exhaustive searching for paths and connections, (ii) a systematic evaluation of the compatibility between the process connectivity matrix and a cause-and-effect matrix derived from process measurements.

\section{The Cause-and-Effect Analyzer}

The outcome of the work reported in this paper is a set of tools which use a CAEX description of the process schematic and measurements from a plant-wide disturbance. The tools generate a causal connectivity matrix to represent the process, and a cause-and-effect matrix showing the empirical relationships between measurement points where the disturbance has been detected.

The cause-and-effect matrix identifies candidate locations for the root cause of the plant-wide disturbance and also gives one or more propagation hypotheses, i.e. which measurement points are affected by secondary propagated disturbances, and in which order (the effects). A search of the process connectivity matrix determines whether a propagation hypothesis is feasible and, if there is more than one hypothesis, it indicates which is the most likely root cause and propagation route.

\subsection{Representation of the process schematic}

The core technology that is used to represent the process schematic of a chemical process is XML. $\mathrm{XML}$ is a scripting language that was developed for the purpose of describing data in a format which ensures that data can be interpreted by both humans and computers. The structure of a CAEX-compliant $\mathrm{XML}$ file for a process schematic is given by a schema which is defined under the Computer Aided Engineering Exchange (CAEX) standard. This schema defines the acceptable entries and format for the XML description. CAEX describes the process schematic as a series of nodes which have standard names and these nodes contain user-defined descriptions of the different elements and the directional connections between them, among other attributes. The availability of a common standard facilitates the development of a generic tool for extraction of a connectivity model from a CAEX description.

The components in the process schematic are entered into specific parts of the XML text file. The node containing the InternalElement element describes each of the items in the process schematic such as pipes and indicators, and the item is described within the start and end tags of the node InternalElement. The text between the smart tags contains the element name which is given by localElementName and also a description given by description which usually explains the function of the item, for example:

$<$ InternalElement

localElementName="Distill-006"

description="Part of Distillation

Column" />

This indicates an element called Distill-006 which is part of a distillation column.

An InternalLink node describes the directional links between the named units. The links are again entered into the file in a format similar to the entries for InternalElement. The name of the link being described is given by the localLinkName description, while refPartnerSideA refers to the unit output from which the link originates and refPartnerSideB refers to the unit input at which the link terminates. These are determined by the direction of flow between the units with the upstream element being entered as refPartnerSideA and the downstream unit being entered as refPartnerSideB, for example:

<InternalLink localLinkName="Link@०3"

refPartnerSideA="Pipe-002:01"

refPartnerSideB $=" F l a s h-001:$ I2" />

This indicates a forward link, named Link003, from the first output (O1) of element Pipe-002 to the second input (I2) of element Flash-001.

3.2 An industrial case study

The process schematic that is used to demonstrate the Cause-and-Effect Analyser tool is shown in Fig. 1 and is the one reported in Bauer et al., (2007). It is a distillation unit operated by the Eastman Chemical Company in Kingsport, Tennessee. The elements described in the CAEX file are labelled in Fig. 2 and include controllers, instruments and signal lines as well as hardware such as pipes.

For the purposes of the connectivity description, the distillation column is partitioned into seven sections. The boundaries between the sections depend on the external connections and their purpose is to reflect the physical layout, for instance that there is a temperature indicator near the top, one in the middle and one near the bottom. It is a similar approach to that used in lumped models of distillation with feed zone, stripping and rectifying sections. The links between the sections of the column are bi-directional because vapour flows up the column and liquid flows down. 




Figure 1. Example process schematic from Bauer et al., 2007 (courtesy of Eastman Chemical Company).

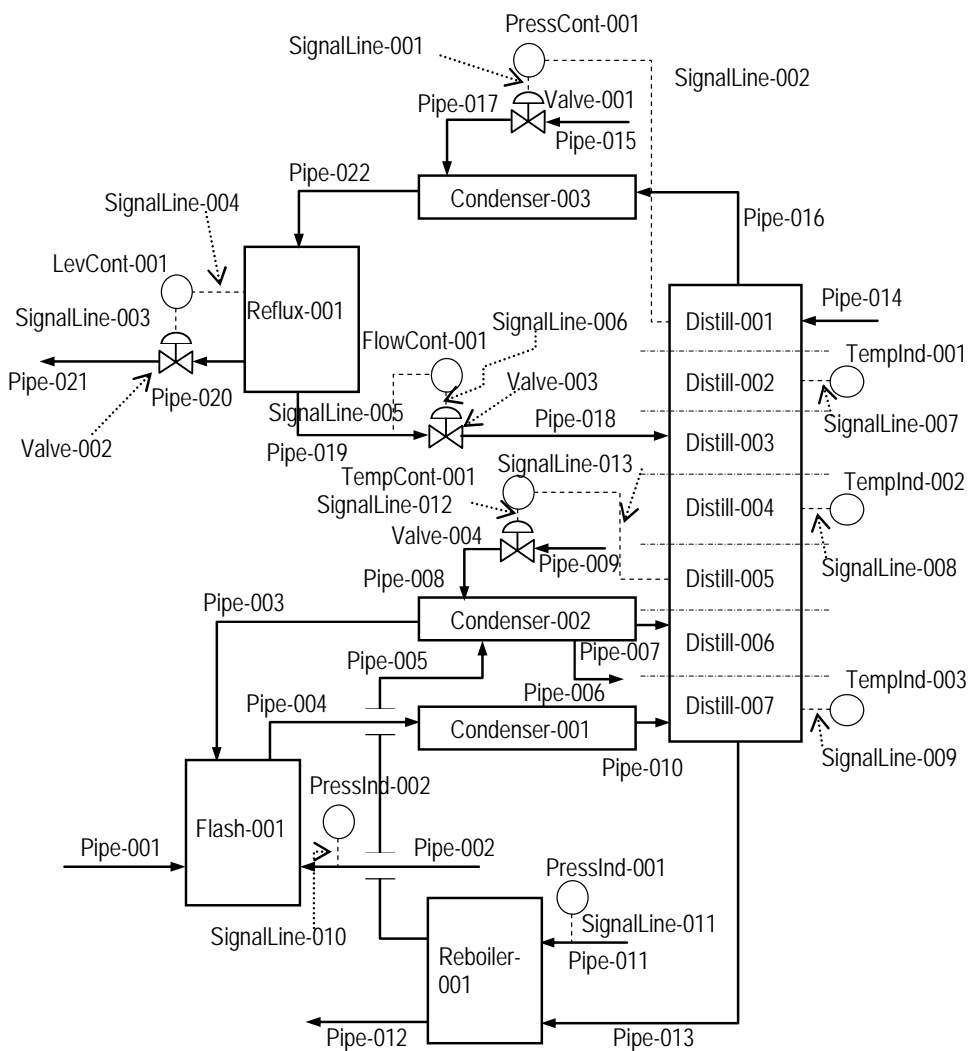

Figure 2. Process schematic with labels for elements

The connectivity representation encoded in the CAEX file reflects the connections of the process equipment as shown in the schematic, but it does not model the mechanisms of the process. For instance, Condenser002 has connections to three pipes conveying process fluids and two conveying utility fluids, but there is no description in CAEX of the interchange of mass or energy between these streams. Clearly, though, physical effects do propagate where there are connections. For instance, flow variations in the cooling water supplied to the condenser cause temperature variations in the process stream entering the distillation column.

\subsection{XML parser and connectivity matrix}

The XML file is read into the diagnostic tool in order to formulate the connectivity model. C\# is used for this purpose. $\mathrm{C \#}$ is an object-oriented programming language that is inherent to the .NET framework, part of the .NET developers' platform that allows the use of different programming languages together. C\# was the language that was developed to run inherently on the framework and it contains functions to read and validate XML files.

In order to extract the information about the process connectivity from the CAEX file, the file is read in a basic fashion using the Read() function in C\# . The Read() function is designed to navigate an XML document from line to line identifying specific node structures and the attributes such as localElementName. This method identifies the specific attributes within the file that are required for the analysis which can then be extracted and stored separately. The parser makes a list of all the elements in the process schematic and the individual connections.

A connectivity matrix is constructed using the parsed information. It has the following features:

- The row and column headers of the matrix contain the names of all the parsed elements as labels.

- The size of the connectivity matrix is $N \times N$ where $N$ is the number of elements.

- The first column lists refPartnerSideA elements from the XML file while the first row lists the refPartnerSideB elements.

- Entries in the matrix take a value of " 1 " or " 0 " depending on whether or not there is a directional link from the row (refPartnerSideA) element to the column (refPartnerSideB) element.

An example of part of the connectivity matrix for the example process in Fig. 1 is shown in Table 1. It shows that there is a forward connection (directional flow) from Condenser-002 to Pipe-003 whereas there is no forward connection from Condenser-002 to Pipe005. These elements can be found in the lower left corner of Fig 2. The connectivity matrix is the adjacency matrix of a directed graph in which the elements are the nodes and the " 1 " entries in the matrix represent the presence of directional arcs between nodes.

Table 1. Part of connectivity matrix

\begin{tabular}{lccc}
$\begin{array}{c}\text { refPartnerSide } B \\
\text { elements } \rightarrow\end{array}$ & Pipe-003 & Pipe-004 & Pipe-005 \\
\hline Flash-001 & 0 & 1 & 0 \\
Reboiler-001 & 0 & 0 & 1 \\
Condenser-002 & 1 & 0 & 0 \\
\hline
\end{tabular}

个refPartnerSideA elements

\subsection{Cause-and-effect matrix}

A cause-and-effect matrix is generated from analysis of process measurements. Any measurement-based method that detects causality could be used to generate the cause-and-effect matrix. As discussed earlier, transfer entropy (Schreiber, 2000) which measures the 
causality between two time series was used for the cause-and-effect analysis in this work. Its application for analysis of process disturbances was reported by Bauer et al., (2007) and it is in commercial use in the Plant Disturbance Analyser module within ABB's Loop Performance Monitor tools (Horch, et al, 2007).

Fig. 3 shows the time series of a disturbance that affected several measurements in the example process (from Bauer et al., 2007), and the cause-and-effect matrix generated by the transfer entropy tools from the measurements is in Table 2. A " 1 " at the intersection of a row and column indicates that transfer entropy found a causal relationship between the measurement point indicated on the left side (the cause) and the measurement point indicated across the top (the effect). The size of the cause-and-effect matrix is $n \times n$ where $n$ is the number of measurement points affected by the plant-wide disturbance.

The cause-and-effect matrix of Table 2 is the adjacency matrix of the directed graph in Fig. 4, in which the nodes that are circled twice indicate possible root causes, and all the nodes appearing in the graph are candidate effect elements since the disturbance has been detected at every point.

The plant-wide disturbance is characterized by irregularly spaced spiky deviations detected at all the measurement points. It is highly likely that there is one root cause disturbance which is propagating around the plant, but the cause-and-effect matrix has suggested three sources. Bauer et al., (2007) resolved the ambiguity by manual inspection of the process schematic. In this paper, the aim is to automate the inspection of the process schematic using the connectivity matrix.

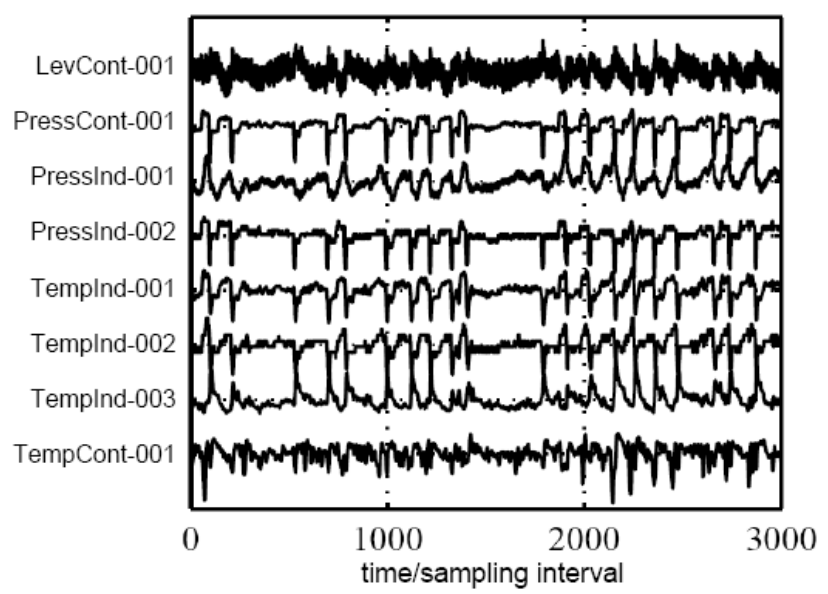

Figure 3: Time series of a plant-wide disturbance, courtesy of Eastman Chemical Company.

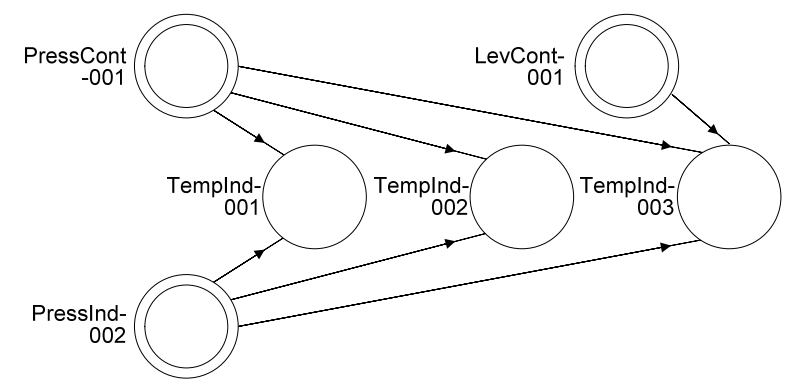

Figure 4. Directed graph generated by the transfer entropy method.
Table 2: Cause-and-effect matrix from transfer entropy

\begin{tabular}{|c|c|c|c|c|c|c|c|c|}
\hline $\begin{array}{r}\text { effect } \\
\text { elements } \\
\rightarrow\end{array}$ & 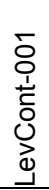 & 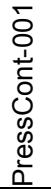 & 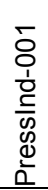 & 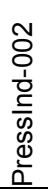 & 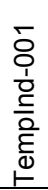 & 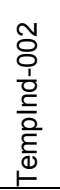 & 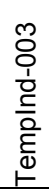 & 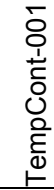 \\
\hline LevCont-001 & - & 0 & 0 & 0 & 0 & 0 & 1 & 0 \\
\hline PressCont-001 & 0 & - & 0 & 0 & 1 & 1 & 1 & 0 \\
\hline PressInd-002 & 0 & 0 & - & 0 & 1 & 1 & 1 & n \\
\hline PressInd-001 & 0 & 0 & 0 & - & 0 & 0 & 0 & 0 \\
\hline Templnd-001 & 0 & 0 & 0 & 0 & - & 0 & 0 & 0 \\
\hline Templnd-002 & 0 & 0 & 0 & 0 & 0 & - & 0 & 0 \\
\hline Templnd-003 & 0 & 0 & 0 & 0 & 0 & 0 & - & 0 \\
\hline TempCont-001 & 0 & 0 & 0 & 0 & 0 & 0 & 0 & - \\
\hline
\end{tabular}

个cause elements

\subsection{Generation of hypotheses}

The cause-and-effect matrix indicates three possible root causes, whereas only one is expected. The reason for this is that the transfer entropy analysis is statistical in nature and there is thus a need to eliminate false positive results which are due to statistical chance. A threshold is set to ensure that transfer entropy detects only the strongest causal relationships. The benefit of setting the threshold high is that it eliminates reporting of spurious relationships, but the penalty is that some weaker causal relationships are overlooked. In this example, transfer entropy did not detect causal relationships which might have resolved the ambiguity between the three postulated root causes: PressInd002, PressCont-001, and LevCont-001. Therefore causal links between these measurements points have to be hypothesised and tested.

The process connectivity matrix allows hypotheses about paths to be tested by checking whether there are feasible propagation paths from the suspected root causes of the disturbance to all the points where the root cause is suspected to have had an effect. The hypothesis that can best explain the propagation to all other points where the disturbance has been measured is the most likely root cause.

\subsection{Search algorithm}

The search algorithm finds feasible propagation paths using the connectivity matrix. The aims are to find all paths starting at the hypothesised root causes, and to test whether there exists a path from each hypothesized root cause element to the other elements where the effects of the disturbance have been observed. The algorithm is based on graph traversal which searches a series of nodes (i.e. the process elements), ensuring that each node is only traversed once in order to ensure that the search is limited from getting into a loop. The particular method used in this technique uses a depthfirst search (Cormen et al., 2001) whereby the search starts from a specified node and then continues exploring along a particular set of nodes until it reaches a termination, at which point it backtracks and continues searching.

The elements in the cause-and-effect matrix are always indicators or controllers because these are the measurement points. The first step in linking of the 
cause-and-effect matrix with the process connectivity matrix is to find the process elements which are attached to the indicators and controllers. It is straightforward to identify these elements without ambiguity since the connection is via a signal line.

The search algorithm starts by moving to the row in the connectivity matrix representing the postulated root cause and then searching for a " 1 " which indicates that the row element is connected to the column element. The search then proceeds to the row representing the column element to find out which element it is connected to and so on, hence compiling all elements in any forward path from the start element using a depth-first search. The termination conditions are (i) that the next element has been visited before, in which case paths from that point are already known, or (ii) that the current element has no refPartnerSideB connection. After one path has terminated, the algorithm backtracks to follow other branches with the same termination conditions. For the generic example in Fig. 5 the compiled list of forward path elements starting from Element-006 is: Element-006, Element005, Element-007, Element-001. An additional connection from Element-007 terminating at Element004 would also be recorded after partial backtracking.

The search then checks whether each element where the disturbance has been detected lies in the compiled list of paths. If it does, then a propagation path exists from the postulated root cause element to a point where the disturbance has been measured. If not, then that root cause hypothesis is not valid since there is no way a disturbance at the postulated root cause could have reached the point of detection. This step establishes that a path exists but does not specify what the path is.

The number of steps in the search has an upper bound of $m \times N$ where $N$ is the number of elements and $m$ is the number of postulated root causes. The reason is that for each postulated root cause the search visits each element at most once.

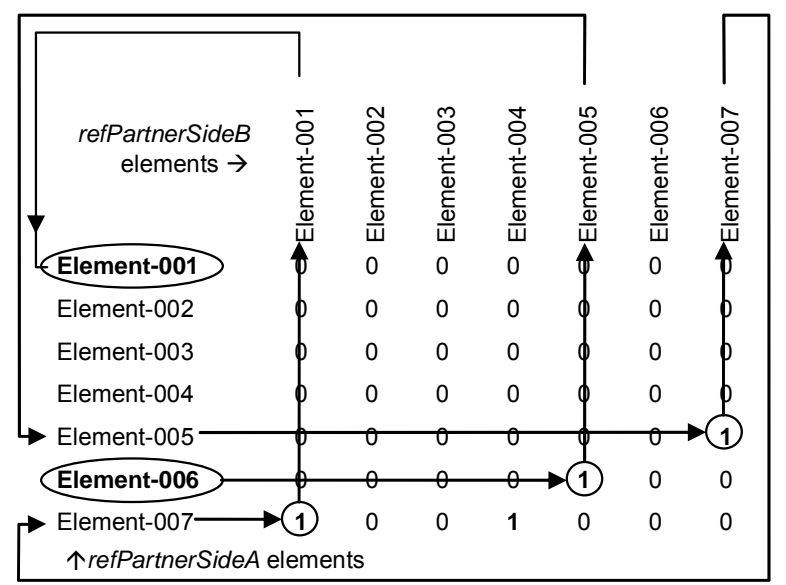

Figure 5: Depth-first search used to compile a list of forward path elements.

\subsection{Determining propagation paths}

The above search compiles a list of all elements in any forward path from the postulated root cause and identifies the postulated root causes for which feasible propagation paths exist to the effect points where the disturbance is being measured. Specific propagation paths are determined using a technique similar to the one used to compile the list of forward path elements. However, in this case, the search starts from a point where the disturbance has manifested (an effect element), and since this represents an end point, the search starts from the column representing the effect element. The search checks the column for a " 1 " which indicates the element to which this column element is connected. It then cross-checks to see whether this element is listed in the list of forward path elements that was compiled using the former depth-first search, because if it is, then the search is moving backwards along a propagation path. In the case that it is, the search moves to the column representing that element and continues the search in a similar manner until the postulated root cause element is reached, thus finding a specific propagation path.

The search is carried out to find paths from all possible effect elements to all the postulated root cause elements. The number of possible propagation paths, including the shortest path and its length are all stored and analyzed later.

If $p$ is the average number of possible paths for each hypothesis then an upper bound for the total number of steps in searching of the connectivity matrix is of the order of $m \times p \times n \times N$, where $m<p<n \ll N$ and $n$ is the number of effect elements at which the disturbance was detected. Parameter $m$ is small because there are typically only a few root cause hypotheses for each disturbance detected in the process. The reason for the $m \times p \times n \times N$ result is that there are $m \times n$ combinations of postulated causes and effects, an average of $p$ paths linking the cause to the effect, and the maximum length of any path is $N$, the number of elements. Since $m<p<n \ll N$, the total number of steps in the search scales roughly linearly with $N$.

Stone and Sipala (1986) gave a theoretical analysis of the growth rate of algorithms for searching of hierarchical trees. Their work found a linear dependence on the depth of the tree for searches having the property that a decision to cut off the search at a node eliminates the entire sub-tree below that node from further consideration. The search of the connectivity matrix can be covered by the same analysis due to the fact that the initial search predetermines the elements in all possible forward paths from each hypothesized cause hence providing a reference list for the cut-off criteria in each subsequent search. Therefore the result of Stone and Sipala gives a theoretical underpinning for having confidence that the size of proposed search of the connectivity matrix will not grow unreasonably for large plants.

\section{Demonstration}

For the example process of Fig. 1, the cause-and-effect matrix in Table 2 and the directed graph in Fig. 4 show that a disturbance at PressCont-001 (the pressure measurement in the distillation column) could be the root cause of the disturbance at TempInd-003 (the temperature at the bottom of the distillation column). Sub-section 4.1 demonstrates the working of the Cause-and-Effect Analyzer to test this hypothesis. A 
full analysis of all the postulated root causes is then executed.

\subsection{Finding paths}

Since PressCont-001 and TempInd-003 are both measurement points, the search algorithm determines which process elements are directly connected to these measurement points. It finds that PressCont-001 is connected to Valve-001 and Distill-001, while TempInd-003 is connected to Distill-007. The search for paths is then carried out from Valve-001 and Distill-001 to Distill-007.

As a demonstration, two of the returned paths from PressCont-001 to TempInd-003 are listed, together with other commentary provided by the analysis tool.

\begin{tabular}{|c|c|}
\hline \multicolumn{2}{|c|}{$\begin{array}{l}\text { CAUSE-EFFECT RELATIONSHIP: } \\
\text { PressCont-001 } \rightarrow \text { Templnd-003 } \\
\text { PressCont-001 is connected to: } \\
\text { Valve-001 } \\
\text { Distill-001 } \\
\text { Templnd-003 is connected to: } \\
\text { Distill-007 } \\
\text { PATHS FROM Valve-001 TO Distill-007 } \\
\text { There are } 6 \text { feasible propagation paths from Valve-001 to } \\
\text { Distill-007 } \\
\text { PATHS FROM Distill-001 TO Distill-007 } \\
\text { There are } 15 \text { feasible propagation paths from Distill-001 to } \\
\text { Distill-007 }\end{array}$} \\
\hline \multicolumn{2}{|c|}{ Selected paths, for demonstration } \\
\hline PATH 1 & PATH 2 \\
\hline Valve-001 & Distill-001 \\
\hline Pipe-017 & Distill-002 \\
\hline Condenser-003 & Distill-003 \\
\hline Pipe-022 & Distill-004 \\
\hline Reflux-001 & Distill-005 \\
\hline Pipe-019 & Distill-006 \\
\hline Valve-003 & Distill-007 \\
\hline \multicolumn{2}{|l|}{ Pipe-018 } \\
\hline \multicolumn{2}{|l|}{ Distill-003 } \\
\hline \multicolumn{2}{|l|}{ Distill-004 } \\
\hline \multicolumn{2}{|l|}{ Distill-005 } \\
\hline \multicolumn{2}{|l|}{ Distill-006 } \\
\hline Distill-007 & \\
\hline
\end{tabular}

\subsection{Testing root cause hypotheses}

Bauer et al., (2007) reported that a physical inspection of the process revealed that the inert gas inflows into Condenser-003 and Flash-001 were coming from the same gas header which was being controlled by a faulty split range pressure controller. Therefore the disturbance enters through Pipe-002 and Pipe-015 and PressInd-002 is the measurement point closest to the root cause.

The upper panel of Fig. 6 presents a screenshot from the Cause-and-Effect Analyser for diagnosis of the root cause of the plant-wide disturbance shown in Fig. 3. The elements in the row across the top of the table are the effect elements, i.e. the measurement points where the disturbance was detected, and the elements in the columns on the left side are the candidate root causes detected by causal analysis of measurements using transfer entropy. Fig 6 shows PressInd-002 is the most promising root cause hypothesis. The steps in the reasoning are as follows:

- Propagation paths exist from PressInd-002 to all the other points in the hypothesis file, including to PressCont-001 and LevCont-001.

- There are no feasible propagation paths from PressCont-001 and LevCont-001 to PressInd-002 which suggests that they cannot explain the occurrence of the disturbance at PressInd-002.

Fig 6. also confirms all the causal links in Table 2 and Fig. 4 that were detected by measurement-based analysis, for instance it shows there are feasible propagation paths from PressCont-001 and PressInd002 to all three of the temperature indicators.

To summarize, the Cause-and-Effect Analyzer confirmed all the causal links indicated by the measurement-based method of transfer entropy, and further indicated that the disturbance at PressInd-002 could not be due to any of the other postulated root causes indicated by transfer entropy analysis. Therefore PressInd-002, which is attached to Pipe-002, is the location where the disturbance originates.

\section{Dealing with multiple causes}

This section demonstrates how the Cause-and-Effect Analyser deals with multiple disturbances. It also demonstrates operation of the Cause-and-Effect Analyzer with more than one method for generation of hypotheses about root causes.

Figure 7 shows the process diagram from the case study reported in Thornhill et. al., (2003a) in which a plant-wide oscillation was detected and diagnosed. The time trends in Fig. 8 show the plant-wide oscillation and also shows there is a second disturbance present in the plant characterized by a sequence of short spiky deviations which affects Tags 23, 24, 25, 26, 27, 28 and 30 in Column 3. Both disturbances are present in Tags 23 to 28. The purpose of this section is to show how measurement-based analysis formulates hypotheses for the cause of each disturbance for testing by the Cause-and-Effect Analyser.

5.1 Detecting disturbances and root causes

Measurement-based analysis was carried out with ABB's Plant Disturbance Analyser tool which includes a number of analysis methods including frequency domain filtering, oscillation detection and clustering (Thornhill et al, 2003b), detection of nonlinear root causes (Thornhill, 2005), transfer entropy (Bauer et al, 2007) and time delay analysis (Bauer, 2008). Measurement-based analysis does most of the hard work of detecting the presence of multiple disturbances and finding the affected measurement points. It also applies root-cause detection methods tailored to the type of disturbance, as discussed in the original references. These are (i) nonlinearity analysis for oscillating disturbances, and (ii) transfer entropy and time delay analysis for non-periodic disturbances. The PDA tool characterized the oscillatory disturbance and the spiky disturbance as two separate effects. It showed the measurement points affected by each disturbance and generated hypotheses about the root causes.

Identification of the root cause from measurementbased analysis is not always conclusive, however, because it may give more than one hypothesis. Nonlinearity analysis suggested Tag 29 (FI3) and Tag 22 (LC2) as possible causes of the plant-wide oscillation, while transfer entropy and time delay analysis both showed Tags 30 (FC7) and 28 (PI2) as possible causes of the disturbance with spiky features. 5.2 Testing root cause hypotheses 
Yim et al (2005) reported previously on the use of a CAEX description of the process to find that control loop LC2 (Tag 22) was upstream of Tag 29 and therefore more likely to be the root cause of the plantwide oscillatory disturbance. The new Cause-andEffect Analyser also pointed to LC2 and confirmed propagation paths to all locations in the plant where the disturbance was detected.

For the disturbance with spiky features, the Cause-andEffect Analyser identified the steam flow controller FC7 (Tag 30) as the root cause. The analyser concluded that pressure indicator PI2 (Tag 28) is affected by the disturbance in the flow controller because PI2 is downstream of the FC7 controller.

\section{Discussion}

XML descriptions of process schematics are a recent development that have not yet received much academic attention, and it therefore seems timely to speculate about developments that would enhance their potential in solving more complicated problems, and to give a critical evaluation of their limitations.

\subsection{Incorporating process information}

The development of CAEX was driven by the needs of engineering contractors to manage and manipulate information about equipment during design, procurement and construction. A CAEX representation of a process schematic includes quantitative information about the elements such as dimensions and materials of construction, but not information about the process itself.

Additional attributes that would be useful are to label the contents of vessels and pipes as liquid, gas, solids or mixed phase, and to label pipes as carriers of utility fluids or of process fluids with the attribute of composition. Such information would allow more subtle analysis such as:

- Analysis of the propagation paths of disturbances that cause variations in (often unmeasured) composition of a process stream. Variations in feed composition can cause variation of temperature in a distillation column, for instance, even if the feed flow rate and temperature are steady.

- Detection of pressure zones that act as a single system. Since pressure variations travel at the speed of sound, a path through a pressure zone could be collapsed to a single step in the propagation analysis. In the example of section 4 , the distillation column would be a pressure zone.

\subsection{Process boundaries.}

The connectivity matrix includes elements that cross the boundaries of the process schematic. They can be identified by their lack of connections, for instance in Fig 2, Pipe-002 (lower left) has just one directional link to Flash-001, but its input is not connected. Pipes crossing the boundary include the utility streams, and the feed, product and inert gas streams also cross the boundary. An automated technique for dealing with connections beyond the boundaries remains to be developed.

The process schematic does not specify what is beyond the boundary, but engineering knowledge could be used to enhance the description. For instance, pipes carrying steam into a local area of the plant are likely to be connected to the same steam header, and thus can be regarded as part of the same pressure zone.

As mentioned earlier, the inert gas flows into Condenser-003 and Flash-001 are from the same gas main and thus, again, can be regarded as part of one upstream pressure zone. Indeed, variability in the pressure in this gas main was found on investigation to be the root cause of the plant-wide disturbance. An upstream pressure zone could be inferred from a CAEX file in which pipes crossing the boundary were classified as carriers of gas, and the possibility could be highlighted to the user. If an upstream gas main were added in the connectivity matrix, then PressInd002 (the confirmed root cause hypothesis) would be interpreted as the pressure of this gas main, which would be a fully insightful result.

This proposed method of dealing with boundaries was simulated by editing the CAEX file manually to add an inert gas main feeding both Pipe-015 and Pipe-002. The lower panel in Fig. 6 shows the path analysis with this manual edit in place. PressInd-002 is again identified as the only root cause hypothesis that explains the disturbance at all other measurement points. The Cause-and-Effect Analyzer also gave the following message specifying the location of the PressInd-002 measurement point:

CAUSE-EFFECT RELATIONSHIP:

Pressind-002 $\rightarrow$ Templnd-003

Pressind-002 is connected to:

InertGasMain-001

Templnd-003 is connected to:

Distill-007

6.3 Evaluation and future development

The advantage of the CAEX-compliant XML representation is the ease with which the description can be generated. On the other hand, while CAEX can generate a directed graph, information about the signs of the influences is not inherent within the description and the representation therefore lacks the predictive power of a first principles model or of a signed digraph. A first principles model may not, however, be available whereas the process schematic is always available. Generation of a CAEX description takes approximately the same length of time as drawing and annotating the process schematic in a CAD tool. By contrast, capture of the schematic is only the first of many steps in a first principles simulation.

An intermediate graphical representation has been proposed by Preisig (2007) who used connectivity information such as is available from CAEX and online data for balancing of mass and energy by a method of agglomeration of the graph to identify sections for which balances can be drawn up. Such an approach might allow the use of mass and energy balances to determine the signs of propagating influences.

A context for the future development of CAEX-based tools lies in research which is building on the CAPEOPEN initiative. CAPE-OPEN provides standardized software interfaces to provide interoperability of commercial flowsheeting tools, unit operation models, physical property packages, and numerical solvers. The COGent environment (Yang et al, 2007) uses software agents to find CAPE-OPEN software components on a local computer, a network, or on the 
Internet that meet the requirements of an engineering task. In a COGent environment, a user of a CAEX representation of a process schematic could discover and examine additional information about the process, for instance a mathematical model of the reaction, equipment models, or basic process knowledge such as the example given earlier that feed composition changes can cause variations in temperature in a distillation column. Such information would enhance the connectivity information by giving the ability to determine the signs of influences propagating through directional links.

\section{Summary and conclusion}

A Cause-and-Effect Analyzer tool has been presented that uses a connectivity matrix to represent the schematic of a chemical process. Using the search method of graph traversal, the matrix can be used to find paths by which disturbances can propagate through the process. Therefore, a generated hypothesis regarding the possible root cause of a plant-wide disturbance may be verified to find a feasible propagation path from the postulated root cause to the point where the disturbance has been measured. An industrial case study showed the developed Cause-andEffect Analyzer gave the same result as a previous manual analysis by experts. The connectivity matrix is therefore shown to be a useful technique of cause and effect analysis.

The connectivity matrix is derived from an XML description of the process schematic according to the Computer Aided Engineering Exchange (CAEX) schema. At this time, as CAEX is becoming established commercially and in the academic literature, it is interesting to consider future developments. Dealing with the boundaries of the process schematic in an intelligent way, and the classification of pipes according to their intended function are two suggestions that would have a useful impact.

\section{Acknowledgements}

The authors thank the Advanced Control Technologies Group at Eastman Chemical Company for providing the industrial case studies. The sponsorship of ABB and the Royal Academy of Engineering for the Research Chair in Process Automation at Imperial College London is gratefully acknowledged, and also a grant to the first author from the Overseas Research Students Awards Scheme (ORS).

\section{References}

Bauer, M., Cox, J.W., Caveness, M.H., Downs, J.J., and Thornhill, N.F., (2007). Finding the direction of disturbance propagation in a chemical process using transfer entropy, IEEE Transactions on Control System Technology, 15, 12-21.

Bauer, M., and Thornhill, N.F., 2008, A practical method for identifying the propagation path of plant-wide disturbances, Journal of Process Control, 18, 707-719.

Blue Circle Industries, plc. (1990). Real time process control: improved efficiency. Expert System Opportunities, Case Study 1, HMSO, London.

Chiang, L.H., and Braatz, R.D., (2003). Process monitoring using causal map and multivariate statistics: fault detection and identification. Chemometrics and Intelligent Laboratory Systems, 65, 159-178.
Cormen, T.H., Leiserson, C.E., Ronald L. Rivest, R.L., and Stein, C., (2001). Introduction to Algorithms, Second Edition. MIT Press and McGraw-Hill, ISBN 0-262-03293-7.

Drath, R.; Fay, A., and Schmidberger, T., (2006), Computer-aided design and implementation of interlock control code, Proc. 2006 IEEE International Symposium on Computer-Aided Control Systems Design, Munich 4-6 Oct. 2006, 2653-2658. DOI: 10.1109/CACSD.2006.285525.

Fedai, M., and Drath, R. (2005). CAEX - A neutral data exchange format for engineering data, ATP International Automation Technology 01/2005, 3, 43-51.

Hetherington, J., Bogle, I.D.L., Saffrey, P., Margoninski, O., Li, L., Varela Rey, M., Yamaji, S., Baigent, S., Ashmore, J., Page, K., Seymour, R.M., A. Finkelstein, A., and Warner, A., (2007), Addressing the challenges of multiscale model management in systems biology, Computers \& Chemical Engineering, 31, 962979.

Holland, P.W., (1986). Statistics and causal inference, Journal of the American Statistical Association, 81, :945-960.

Horch, A., Cox, J., and Bonavita, N., (2007). Peak performance: Root cause analysis of plant-wide disturbances, $A B B$ Review 1/2007, 24-29.

Lam, C.P., Li, H., and Xu, D., (2007), A model-centric approach for the management of model evolution in chemical process modelling, Computers \& Chemical Engineering, 31, 1633-1662.

Larsson, J.E., (2007). GoalArt, Knowledge for Safe Operation, online: http://www.goalart.com/, retrieved 26 August 2008.

Laser, M., (2000). Recent safety and environmental legislation. TransIChemE B: Process Safety and Environmental Protection, 78, 419-422.

Laud, A., (2006). XMpLant Model Overview, online: http://www.noumenon.co.uk/XMpLant model overview.pdf retrieved 26 August 2008.

Leal, D., (2005). ISO 15926 "Life Cycle Data for Process Plant": An overview, Oil \& Gas Science and Technology -Rev. IFP, 60, 629637.

Lee, G.B, Song, S.O., and Yoon, E.S., (2003). Multiple-fault diagnosis based on system decomposition and dynamic PLS, Industrial \& Engineering Chemistry Research, 42, 6145-6154.

Leung, D., and Romagnoli, J. (2002). An integrated mechanism for multivariate knowledge-based fault diagnosis, Journal of Process Control, 12, 15-26.

Maurya MR, Rengaswamy R, Venkatasubramanian V., (2003a), A systematic framework for the development and analysis of signed digraphs for chemical processes. 1. Algorithms and analysis, Industrial \& Engineering Chemistry Research, 42, 4789-4810.

Maurya MR, Rengaswamy R, Venkatasubramanian V, (2003b), A systematic framework for the development and analysis of signed digraphs for chemical processes. 2. Control loops and flowsheet analysis, Industrial \& Engineering Chemistry Research, 42, 48114827.

Maurya, M.R., Rengaswamy, R. and Venkatasubramanian, V., (2004). Application of signed digraphs-based analysis for fault diagnosis of chemical process flowsheets. Engineering Applications of Artificial Intelligence. 17, 501-518.

Maurya MR, Rengaswamy R, Venkatasubramanian V., (2007), A signed directed graph and qualitative trend analysis-based framework for incipient fault diagnosis, Chemical Engineering Research \& Design, 85, 1407-1422.

Nimmo, I. (1995), Adequately address abnormal situation operations. Chemical Engineering Progress, 91, 36-45.

Norvilas A, Negiz A, DeCicco J, Cinar A., (2000). Intelligent process monitoring by interfacing knowledge-based systems and multivariate statistical monitoring, Journal of Process Control, 10, 341-350.

Palmer, M., (2007). Automating Equipment Information Exchange $(A E X)$, online: http://www.fiatech.org/projects/idim/aex.htm, retrieved 26 August 2008.

Pearl, J., (1995). Causal diagrams for empirical research, Biometrika, 82, 669-688.

Petersen, J., (2000). Causal reasoning based on MFM. Proceedings of Cognitive Systems Engineering in Process Control (CSEPC 2000), Taejon, Korea, 36-43.

Preisig, H.A., (2007). A graph-theory-base approach to the analysis of large-scale plants, Proc. 17th European Symposium on Computer Aided Process Engineering - ESCAPE17, 81-86.

Schmidberger, T., Horch, A., Fay, A., Drath, R., Rule based engineering of asset management system functionality. Proceedings of 5th Vienna Symposium on Mathematical Modelling, February 7 - 10, 2003, pp 2.1 - 2.8.

Schreiber, T., (2000), Measuring information transfer, Physical Review Letters, 85, 461-464. 
Srinivasan, R., Maurya, M.R., and Rengaswamy, R., (2006). Root cause analysis of oscillating control loops, Proceedings of IFACADCHEM 2006, Gramado, Brazil, April 3-5.

Stone, H.S., and Sipala, P., (1986), The average complexity of depth-first search with backtracking and cutoff, IBM Journal of Research and Development, 30, 242-258.

Thornhill, N.F., Cox, J.W., and Paulonis, M., (2003a). Diagnosis of plant-wide oscillation through data-driven analysis and process understanding, Control Engineering Practice, 11, 1481-1490.

Thornhill, N.F., Huang, B., and Zhang, H., (2003b). Detection of multiple oscillations in control loops, Journal of Process Control, 13, 91-100.

Thornhill, N.F., (2005). Finding the source of nonlinearity in a process with plant-wide oscillation, IEEE Transactions on Control System Technology, 13, 434-443.

Varma, V.A., JPekny, J.F., Reklaitis, G.V., and Subramanian, D., (2003). An XML-based language for the Research \& Development pipeline management problem, Computers \& Chemical Engineering, 27, 1361-1379.

Vedam H, Venkatasubramanian V., (1999). PCA-SDG based process monitoring and fault diagnosis, Control Engineering Practice, 7, 903-917.

Venkatasubramanian, V., Rengaswamy, R., Yin, K., and Kavuri, S.N., (2003a). A review of process fault detection and diagnosis. Part I: Quantitative model-based methods. Computers \& Chemical Engineering, 56, 293-311.
Venkatasubramanian, V., Rengaswamy, R., and Kavuri, S.N., $(2003 b)$. A review of process fault detection and diagnosis. Part II: Qualitative models and search strategies. Computers \& Chemical Engineering, 56, 313-326.

Venkatasubramanian, V., Rengaswamy, R., Kavuri, S.N., and Yin, K., (2003c). A review of process fault detection and diagnosis. Part III: Process history based methods. Computers \& Chemical Engineering, 56, 327-346.

Vesely, W.E.; Goldberg, F.F., Roberts, N. H., Haasl, D.F., (1981). Fault Tree Handbook, U.S. Nuclear Regulatory Commission, NUREG-0492.

Weidl, G., Madsen, A.L., and Israelson, S., (2005). Applications of object-oriented Bayesian networks for condition monitoring, root cause analysis and decision support on operation of complex continuous processes, Computers \& Chemical Engineering. 29, 1996-2009.

Yang, A., Braunschweig, B. Fraga, E.S., Guessoum, Z., Marquardt, W., Nadjemi, O., Paen, D., Piñol, D., Roux, P., Sama, S., Serra M., and Stalker I., (2007), A multi-agent system to facilitate component-based process modelling and design, Computers \& Chemical Engineering, in press, doi: 10.1016/j.compchemeng.2007.11.005.

Yim, S.Y., Ananthakumar, H.G., Benabbas, L., Horch, A., Drath, R., and Thornhill, N.F., (2006). Using process topology in plant-wide control loop performance assessment, Computers \& Chemical Engineering, 31, 86-99.

Proposed Effect Element

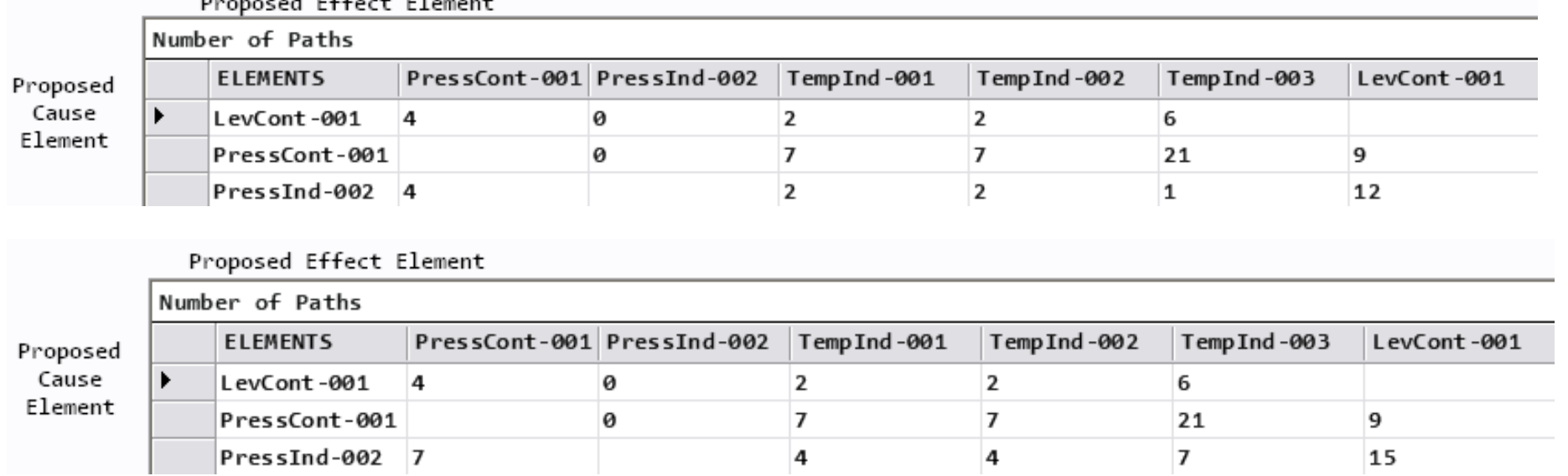

Figure 6. Output of the Cause-and-Effect Analyzer for the example process. Upper panel: Output using the CAEX representation of the process. Lower panel: Output after an upstream inert gas main is added manually. 


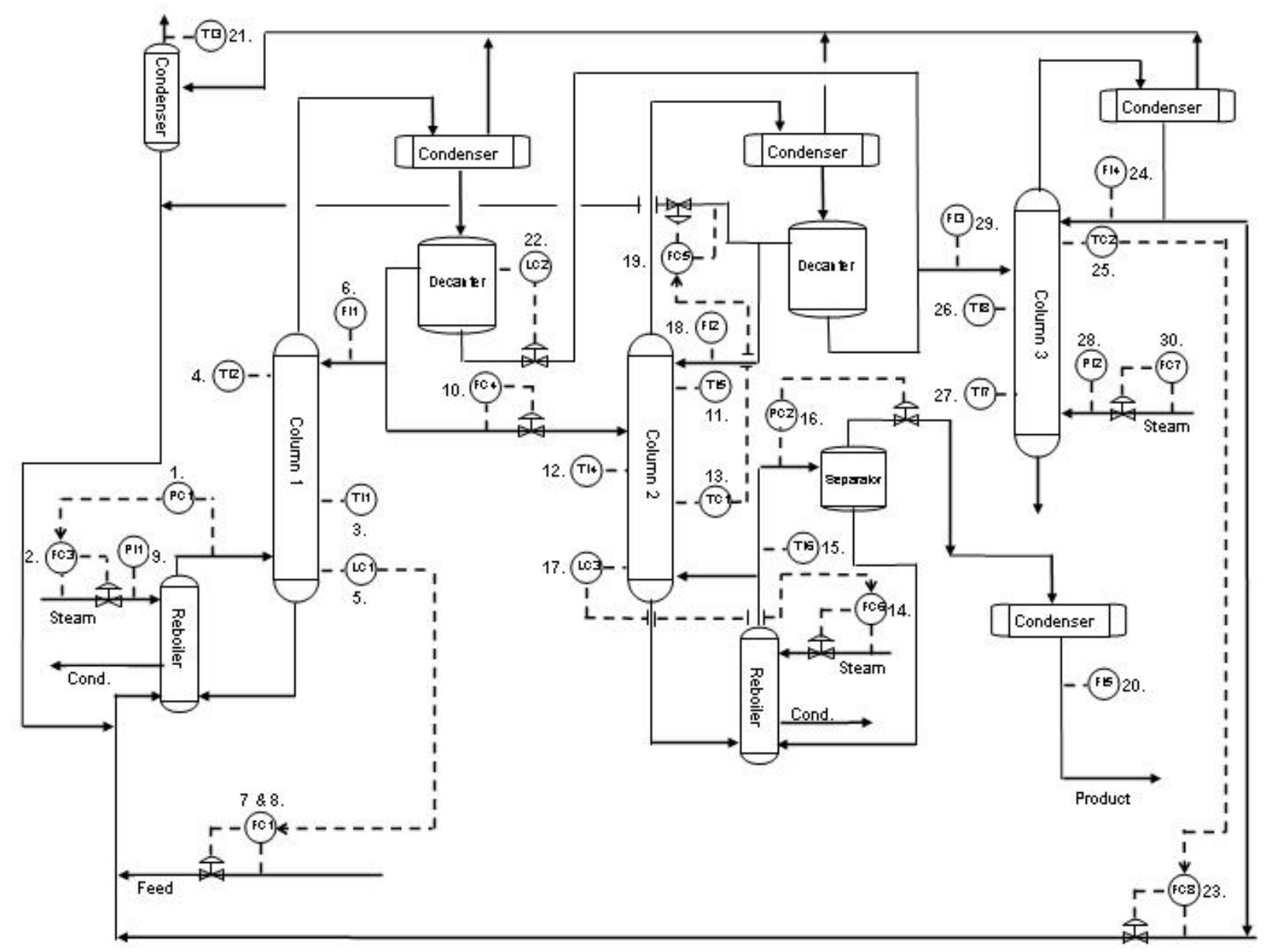

Figure 7. Process schematic from Thornhill et al., 2003a (courtesy of Eastman Chemical Company).
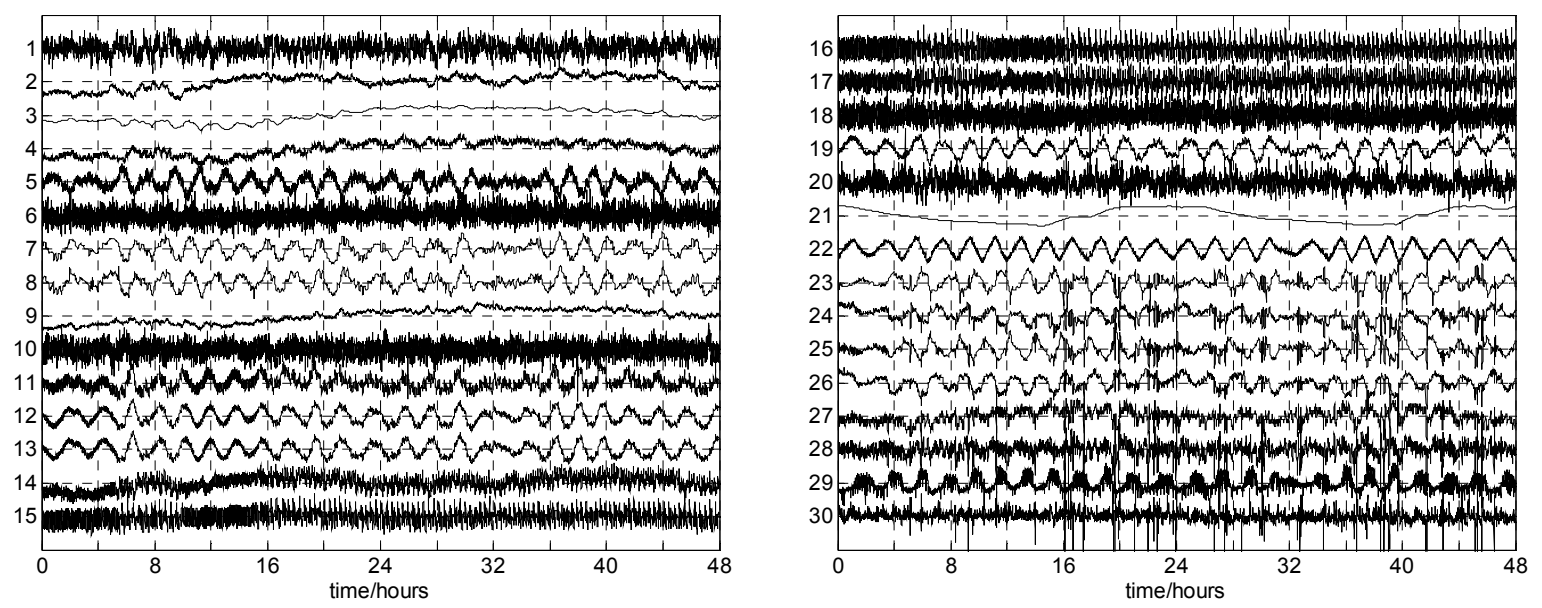

Figure 8. Time series of plant-wide disturbances in the process of Figure 7 showing multiple disturbances (courtesy of Eastman Chemical Company). 